\title{
The lung microbiome in health and disease
}

\author{
Authors: Miriam F Moffatt ${ }^{A}$ and William OCM Cookson ${ }^{B}$
}

The Human Microbiome Project began 10 years ago, leading to a significant growth in understanding of the role the human microbiome plays in health and disease. In this article, we explain with an emphasis on the lung, the origins of microbiome research. We discuss how $16 \mathrm{~S}$ rRNA gene sequencing became the first major molecular tool to examine the bacterial communities present within the human body. We highlight the pitfalls of molecular-based studies, such as false findings resulting from contamination, and the limitations of 16S rRNA gene sequencing. Knowledge about the lung microbiome has evolved from initial scepticism to the realisation that it might have a significant influence on many illnesses. We also discuss the lung microbiome in the context of disease by giving examples of important respiratory conditions. In addition, we draw attention to the challenges for metagenomic studies of respiratory samples and the importance of systematic bacterial isolation to enable host-microbiome interactions to be understood. We conclude by discussing how knowledge of the lung microbiome impacts current clinical diagnostics.

\section{Introduction}

Understanding the human microbiota has the potential to offer better diagnosis and rational management of many human diseases. Thus, it is not surprising that the scientific and lay press have embraced the human microbiome over the past decade. Before turning to the lung, it is worthwhile understanding the origins and meaning of 'microbiome'.

The Oxford English Dictionary online tells us the term 'microbiome' first appeared in a 1952 publication discussing water pollution by sewage. ${ }^{1}$ Joshua Lederberg, awarded a Nobel prize for his work on microbial genetics, proposed in 2001 that 'microbiome' be used to describe 'the collective genomes of all the microorganisms inhabiting a specific environment, especially that of the body'?

The definition of 'microbiome' now includes every organism (not only bacteria, but also Archaea, fungi and viruses) and every means of accessing its DNA (metagenomics), its metabolites, RNA species and proteins, ${ }^{3}$ in recognition of the central role it has in human health and disease. The Human

Authors: A professor of respiratory genetics, National Heart and Lung Institute, Imperial College London, UK; ${ }^{B}$ professor of genomic medicine, National Heart and Lung Institute, Imperial College London, UK
Microbiome Project (HMP) was initiated in 2007 by the US National Institutes of Health, ${ }^{4}$ with a consequent flood in human microbiome studies.

A fundamental goal of the HMP was to identify and characterise the microbiome at key sites throughout the human body. Here, we examine how the technology arising from the HMP is applicable to the lung disorders that commonly affect much of humanity.

The first major tool in this effort has been the application of $16 \mathrm{~S}$ rRNA gene sequencing. More advanced approaches, such as whole-genome sequencing and metagenomics, have been applied to tuberculosis and more tentatively to other lung diseases and are described towards the end of this review.

\section{S rRNA gene sequencing}

16S rRNA gene sequencing is an easily applicable technology that gives a description of the bacteria present in a complex biological mixture, allowing investigation of whole communities and the identities of their constituent members.

Bacteria are traditionally classified taxonomically into phyla, genera and species based on differences in phenotypic characteristics, and phenotypic tests still underpin much of standard clinical microbiology. Genetic sequence variation provides a more-robust means of tracing the evolution of organisms from the most simple to the most complex. Carl Woese and others during the late 1970s pioneered sequencing of the small subunit ribosomal (16S rRNA) gene ( $r r n A)$ to address bacterial phylogeny. ${ }^{5,6}$ This gene was particularly suitable because ribosomes are a conserved component of the transcriptional apparatus of all DNA-based life forms.

The gene comprises nine constant regions (C) and nine hypervariable regions (V1-V9). ${ }^{7}$ The variable regions enable sequence-specific discrimination between different bacteria. Using primers to span hypervariable regions, the coupling of $16 \mathrm{~S}$ rRNA polymerase chain reaction (PCR) with nextgeneration sequencing (NGS) of amplicons makes it feasible to sequence many samples at low cost. The sequences amplified can be identified by mapping to reference databases, thereby providing semiquantitative estimates of the bacteria present in any sample. ${ }^{7}$ Careful PCR primer design is essential to control biases for and against particular groups of organism. ${ }^{8}$

Amplicon sequences often do not map directly to classically determined taxonomies or species; thus, clusters of similar sequences are referred to as 'operational taxonomic units' (OTUs). ${ }^{9}$ This limitation of $16 \mathrm{~S}$ rRNA amplicon sequencing is important, in that most bacteria will only be identified at the genus or family level and extremely rarely at the species level, 
depending on amplicon targeted. ${ }^{10}$ NGS approaches focused on variation in other genes might be able to address these limitations.

$16 \mathrm{~S}$ rRNA gene sequences are widely used in environmental studies, where parameters, such as diversity, richness and evenness, are used as measures of the health and resilience of the whole microbial community. ${ }^{9}$ Although these statistics are also applied to human microbial communities, they are probably less informative clinically than knowing the numbers of particular bacterial species and to what extent pathogens dominate the commensals.

Bacteria are ubiquitous, living on all our epithelial surfaces as well as our bowels and they are in rich abundance in most environments. As a consequence, molecular microbiome studies need to minimise the risk of contamination and false positive findings. Contamination can occur at the time of sampling, at DNA extraction and during the downstream steps of PCR amplification and sequencing. ${ }^{11,12}$ Samples from the lung can contain relatively few bacteria (referred to as low biomass), ${ }^{13}$ in contrast to faecal samples, and inclusion of extensive controls against contamination at many levels of the process is advisable.

\section{Are the lungs sterile?}

When the HMP was initiated, the lungs and airways were omitted from the list of organs to be studied. This reflected the common belief that healthy lungs were sterile and perhaps because access to the lower airways was difficult without invasive procedures, such as bronchoscopy. ${ }^{14}$ This oversight has delayed the systematic investigation of the lung microbiome.

The first report that the lower airways carried a similar bacterial density as the upper small bowel ${ }^{15}$ was greeted with scepticism, with a primary concern that the results reflected contamination from passage of the bronchoscope through the upper respiratory tract. However, the use of bronchoscopy samples for investigation of the lung microbiome has been validated by careful studies of the bacterial topography in the healthy human airways. ${ }^{13,16,17}$

The existence of a healthy lung microbiome is now generally accepted, together with recognition that its disturbances influence many lung diseases. There is consensus from $16 \mathrm{~S}$ rRNA studies that, at the family level, the healthy lung is typified by the presence of Firmicutes, Bacteriodetes, Proteobacteria, Fusobacteria and Actinobacteria, while, at the OTU level, Prevotella, Veillonella and Streptococcus spp. are characteristic. $^{13,16-18}$ In common with other mucosal surfaces, many of the consistently observed OTUs are facultative anaerobes. Anaerobic incubation is not a routine component of standard clinical microbiology for sputum, perhaps explaining the early presumption that normal healthy lungs were sterile.

Bronchoscopy requires careful monitoring of patients for several hours and, although it is safe in a clinical setting (including clinical trials), it has not been attempted in representative population samples. In health, the oropharynx and intrathoracic airways might carry very similar microbiota $^{15,16}$ (although nasal swabs do not reflect the lower airway microbiome ${ }^{17}$ ) and oropharyngeal swabs might be useful epidemiologically. However, in disease states, the oropharynx does not consistently match the lower airways ${ }^{15}$ and regional differences might be present within the lung itself. ${ }^{17}$ Sputum is used clinically as an effective surrogate for airway samples, despite acknowledged contamination from upper airways and saliva. In the absence of sputum production, a reliable means of lower airway sampling remains an obstacle to large-scale investigations of lung disease or serial studies requiring frequent sampling.

\section{Respiratory diseases}

\section{Asthma and chronic obstructive pulmonary disease}

Asthma is characterised by abnormal airway mucosa, inflammation and intermittent wheeze. In westernised populations, asthma is the most common chronic illness of childhood. It is estimated to affect 334 million people ${ }^{19}$ worldwide, with an economic burden of $€ 20$ billion each year in just the European population. ${ }^{20}$ Viral-induced exacerbations are the major cause of morbidity in childhood asthma. ${ }^{21}$ There have been consistent epidemiological indications of the importance of the microbiome in asthma, dating back to David Strachan's 'hygiene hypothesis', which he invoked to explain why youngest children had less atopy than their older siblings. More recently, the PARSIFAL and GABRIELA studies have shown that exposure to a rich microbial environment early in life, such as growing up on a farm, can protect against asthma development. $^{22}$

A 16S microbiome study in 2010 compared bronchial brushings from asthmatic and healthy lungs ${ }^{15}$ and showed for the first time that the bronchial tree contained a characteristic flora. In patients with asthma, the microbiota were perturbed by the presence of known and potential pathogens, such as Haemophilus and Neisseria spp. (members of the Proteobacteria), accompanied by reductions in the numbers of commensals, such as Prevotella and Veillonella spp. This characteristic signature has been confirmed by others ${ }^{18,23}$ and has led to the concept that forms of dysbiosis underlie asthma. ${ }^{24}$

Chronic obstructive pulmonary disease (COPD) currently affects 64 million people worldwide. It is characterised by inflammation of the small airways that intensifies as the disease progresses. Although smoking is recognised to be a primary factor, not all smokers develop the disease and the disease continues to progress after smoking cessation. ${ }^{25}$ Exacerbations resulting from recurrent infections are associated with accelerated decline of lung function and worsened quality of life and poor prognosis. As with asthma, Hilty et al. showed Proteobacteria to be increased in COPD airways, in keeping with numerous studies identifying Haemophilus influenzae as an important pathogen in COPD exacerbations. ${ }^{15}$ Replication of these findings has been variable and somewhat influenced by sample type (bronchial brush, bronchoalveolar lavage, endotracheal aspirates or lung tissue). ${ }^{25}$ It has been proposed that differences between studies reflect the existence of more than one microbial profile for COPD, with different phyla dominating, ie Proteobacteria versus Firmcutes. ${ }^{25}$

These cross-sectional investigations have only looked at samples taken at a single time point. Consequently, there is the need for longitudinal studies, where an individual patient can act as their own control, to establish the presence or absence of a causal link between microbiota and disease. Such studies allow alterations in the airway microbiome because of 
treatment and exacerbation to be monitored by repeat sampling over a period of time.

\section{Chronic suppurative lung diseases}

Chronic suppurative lung disease (CSLD) encompasses conditions characterised by progressive lung damage and a chronic productive cough. Two major diseases that come under this banner are cystic fibrosis (CF) and non-CF bronchiectasis (BX). Both feature chronic airway infections that bring significant morbidity, and a very high mortality in the case of CF. There has been considerable interest in bacterial characterisation using $16 \mathrm{~S}$ approaches, initially using $16 \mathrm{~S}$ rRNA gene-based microarrays and more recently using NGS.

Individuals with CF have long been recognised by clinical culture to harbour key pathogens that include Pseudomonas aeruginosa and a Burkholderia cepacia complex. ${ }^{26}$ Although molecular-based methods confirmed the presence of these taxa in adults with CF, they revealed that the lower airway contents in CF are more polymicrobial than previously thought. ${ }^{27}$ Studies of paediatric patients with CF have been more challenging because lower airway sampling is not part of standard clinical care in this age group and many children with CF do not naturally expectorate sputum.

Three longitudinal studies for CF have been published, varying in length and whether treatment of exacerbations was considered. ${ }^{28-30}$ The longest study followed patients for a year with home sampling of sputum three times a week. ${ }^{28}$ Although only six patients were studied, changes in the microbial community structure were not unique to exacerbations but were observed during clinical stability. An important finding was that the lung microbiome was patient specific, such that diversity among subjects was often tightly correlated to an individual rather than shared among groups of individuals.

Bronchiectasis is characterised by abnormal dilated thickwalled bronchi and purulent sputum. Exacerbations are common and an underlying assumption is that they are driven by the overgrowth of a particular microbial species. The only longitudinal study to date has been conducted over 6 months with sputum samples collected monthly and during periods of exacerbation. The microbiota was quantified by $16 \mathrm{~S}$ rRNA gene sequencing. ${ }^{31}$ Similar to the findings for CF, the subjects were found to have a more complex airway microbiome than that revealed by standard clinical culture. The microbial communities were highly individual in composition and stability and there was a poor correlation with clinical state.

\section{Host factors}

For nearly all the lung diseases mentioned above, there has been limited consideration of the genetic background of the host (us, the human) and how this interplays with the airway microbiome. For CF, there are more than 2,000 known mutations of the gene encoding CF transmembrane conductance regulator (CFTR), making stratification of microbial findings by genotype other than $\Delta 508$ daunting. Of interest is that restitution of CFTR function with ivacaftor modified the microbiome, presumably for the better. ${ }^{32}$ For idiopathic pulmonary fibrosis (IPF), the most-common mutation in the gene encoding Mucin 5B
$(M u c 5 B)$ was found to be associated with the total levels (burden) of bacteria present. ${ }^{33}$ More interesting perhaps is the recent study of the Fucosyltransferase 2 (FUT2) genotype in non-CF bronchiectasis and its impact on P. aeruginosa dominance of the microbiota present. ${ }^{34}$ For asthma, it is notable that the genetic risk factors identified by genomewide association studies are expressed by or within the respiratory epithelium, perhaps reflecting the importance of the respiratory microbiome. ${ }^{35}$ Understanding more about host-microbiome interactions could follow from comparing gene expression in airway samples to microbial communities in various diseases.

\section{Metagenomics}

Almost all respiratory microbiome studies to date have relied only on sequencing the $16 \mathrm{~S}$ rRNA gene. However, bacterial genome sequencing has highlighted that, although a 'typical' bacterium has 5,000 genes, this varies pending on genome size, although coding density (ie number of genes per kilobase (kb) of DNA) is fairly consistent. ${ }^{36}$ Most bacterial genomes exist as a single circular chromosome, although species with linear chromosomes are recognised. ${ }^{37}$ Bacteria additionally contain plasmids (extrachromosomal DNA), the regulation and replication of which is independent of the chromosomes. Many antibiotic resistance genes as well as virulence factors are contained within these plasmids. ${ }^{38}$ Bacteria are also adept at exchanging genetic information through transduction, conjugation and competence. ${ }^{39}$

The metagenome is 'the collection of genomes and genes from the members of a microbiota', and metagenomics is 'the process to characterize the metagenome, from which information on the potential function of the microbiota can be gained'. ${ }^{3}$ The principle of metagenomics is to extract DNA from a complex microbial community and then to shotgun sequence and interpret the data as a single genome. Function for the whole community can then be inferred by the presence of particular classes of genes (such as antibiotic resistance, metabolism and virulence).

Metagenomics has added greatly to understanding of the bowel microbiota and concurrent systematic isolation and sequencing of gut bacteria is allowing genome assembly of specific taxa from the metagenomic shotgun sequences (Human Pan-Microbe Communities (HPMC) database ${ }^{40}$ ). However, the application of metagenomics to the respiratory field is in its infancy.

A substantial hurdle to respiratory metagenomics is that the human DNA:microbial DNA ratio might be very high. For faecal samples, the converse is true. Lung metagenomic studies to date have used sputum samples ${ }^{41}$ and have necessitated a depth of sequencing that makes large-scale studies involving low-biomass samples (such as oropharyngeal swabs) unfeasible and costly. For metagenomics to work in the lung, robust methods that deplete human DNA or enrich microbial genomes are needed before sequencing is carried out.

Methods exist for inferring gene content from public databases, accessing bacterial genomes by looking up $16 \mathrm{~S}$ OTU sequences (eg the PICRUst program ${ }^{42}$ ). This is effective only so far as the OTU sequences closely identify the bacterial species (which is not yet the case) and the public databases comprehensively contain the genome sequences. 
Inferring gene content could be made more accurate by the systematic isolation and sequencing of individual bacterial isolates into a lung culture collection. This approach is exemplified by culture collections for the bowel ${ }^{43}$ and oral cavity. ${ }^{44}$ A lung culture collection appears fundamental for successful genome assemblies and for downstream functional experiments, and should be a priority for lung microbiome research.

\section{Mechanisms of disease}

Although profound, the differences in the airway microbiome between health and disease do not on their own discriminate between cause and effect. It is possible, for example, that Proteobacteria dominating the airway microbiota of patients with asthma or COPD might be secondary to treatment with inhaled steroids. Clinical studies can be designed to control for treatment effects, but a more direct way to understand what bacteria might be doing is to study isolated potential pathogens and their interactions with commensals in vitro using cellular models of the airway mucosa.

An unbiased sequenced-based approach to investigate host effects is to use metatranscriptomics, ${ }^{3}$ which is the analysis of all expressed RNA present in a sample. This has the potential to inform gene expression in the host as well as the microbial community in vivo. Application of this to clinical samples, such as sputum, is very much in its infancy and, as with metagenomics, there are considerable technical and analytical hurdles to be overcome.

\section{Clinical diagnostics}

The insights gained so far into the respiratory microbiome in diseased states have led to considerable interest in the potential development of biomarkers, improved diagnostics and therapies. However, as highlighted above, most respiratory microbiome studies are exploratory and limited in their explanation of causal mechanisms. Microorganism colonisation advantages, virulence factors and antibiotic resistance profiles are poorly characterised because of difficulties with metagenomic techniques.

16S rRNA and other gene sequences from bacterial isolates can be a powerful adjunct to culture for microbial identification. ${ }^{45}$ It is possible for $16 \mathrm{~S}$ rRNA gene analysis of the whole bacterial community from biological samples, such as sputum and bronchoalveolar lavage (BAL) to detect pathogens without the need for culture. However, many lung pathogens (a pathogen is 'a microbe that can cause disease $^{\text {s6 }}$ ), such as Streptococcus pneumoniae and $H$. influenza, are carried in the healthy population and the factors that cause the switch between carriage and disease (such as inhibition by commensal bacteria or intercurrent viral infections) are only partly understood. ${ }^{47}$ Therefore, criteria other than a simple presence or absence of the pathogen are necessary to define a true infection. Part of this process might be estimates of the total bacterial burden present in a sample $e^{33}$ and whether the pathogens dominate the commensal community.

Moving further forward, it is possible to use sequences to quantify the number and type of antimicrobial resistance genes and eventually to identify specific adhesion or virulence factors.
Although direct sequencing, PCR assays for particular genes or microbial identifiers, and proteomics are certain to have a major impact on clinical microbiology, they are unlikely to completely replace standard microbial cultures, where costeffectiveness versus clinical benefit is a key consideration. ${ }^{48}$ Most likely is a progressive co-development of culture and sequence, with great eventual benefit to patients with lung diseases.

\section{Conclusions}

In this review, we have provided a chronological account of what is currently known about the bacterial element of the lung microbiota. The likely complex interactions between bacteria, viruses and fungi should be considered in future research. The advanced technologies of the HMP offer huge opportunities to improve the management of many lung diseases. The challenge is to frame studies that will provide clear guidance for the pressing clinical problems that attend pulmonary microbial disorders.

\section{References}

1 Mohr JL. Protozoa as indicators of pollution. Scientific Monthly 1952;74:7-9.

2 www.oed.com [Accessed 10 October 2017].

3 Marchesi RJ, Ravel J. The vocabulary of microbiome research: a proposal. Microbiome 2015;3:31.

4 Turnbaugh PJ, Ley RE, Hamady M et al. The human microbiome project. Nature 2007;449:804-10.

5 Fox GE, Magrum LJ, Balch WE, Wolfe RS, Woese CR. Classification of methanogenic bacteria by $16 \mathrm{~S}$ ribosomal RNA characterization. Proc Natl Acad Sci USA 1977;74:4537-41.

6 Woese CR, Fox GE. Phylogenetic structure of the prokaryotic domain: the primary kingdoms. Proc Natl Acad Sci USA 1977;74:5088-90.

7 Chakravorty S, Helb D, Burday M, Connell N, Alland D. A detailed analysis of $16 \mathrm{~S}$ ribosomal RNA gene segments for the diagnosis of pathogenic bacteria. J Microbiol Methods 2007;69:330-9.

8 Klindworth A, Pruesse E, Schweer T et al. Evaluation of general 16S ribosomal RNA gene PCR primers for classical and next-generation sequencing-based diversity studies. Nucleic Acids Res 2013;41:e1.

9 Navas-Molina JA, Peralta-Sánchez JM, González A et al. Advancing our understanding of the human microbiome using QIIME. Methods Enzymol 2013;531:371-444.

10 Molyneaux PL, Mallia P, Cox MJ et al. Outgrowth of the bacterial airway microbiome after rhinovirus exacerbation of chronic obstructive pulmonary disease. Am J Respir Crit Care Med 2013; 188:1224-31.

11 Tanner MA, Goebel BM, Dojka MA, Pace NR. Specific ribosomal DNA sequences from diverse environmental settings correlate with experimental contaminants. Appl Environ Microbiol 1998;64:311030.

12 Salter SJ, Cox MJ, Turek EM et al. Reagent and laboratory contamination can critically impact sequence-based microbiome analyses. BMC Biol 2014;12:87.

13 Segal LN, Alekseyenko AV, Clemente JC et al. Enrichment of lung microbiome with supraglottic taxa is associated with increased pulmonary inflammation. Microbiome 2013;1:19.

14 Dickson RP, Erb-Downward JR, Huffnagle GB. The role of the bacterial microbiome in lung disease. Expert Rev Respir Med 2013;7:245-57.

15 Hilty M, Burke C, Pedro H et al. Disordered microbial communities in asthmatic airways. PLoS ONE 2010;5:e8578. 
16 Charlson ES, Bittinger K, Haas AR et al. Topographical continuity of bacterial populations in the healthy human respiratory tract. Am J Respir Crit Care Med 2011;184:957-63.

17 Dickson RP, Erb-Downward JR, Freeman CM et al. Bacterial topography of the healthy human lower respiratory tract. MBio 2017;8:e2287-16.

18 Cardenas PA, Cooper PJ, Cox MJ et al. Upper airways microbiota in antibiotic-naïve wheezing and healthy infants from the tropics of rural Ecuador. PLoS ONE 2012;7:e46803.

19 Ganaie MB, Munavvar M, Gordon M, Lim HF, Evans DJ. Patientand parent-initiated oral steroids for asthma exacerbations. Cochrane Database Syst Rev 2016;12:CD012195.

20 Accordini S, Corsico AG, Braggion M et al. The cost of persistent asthma in Europe: an international population-based study in adults. Int Arch Allergy Immunol 2013;160:93-101.

21 Jackson DJ, Johnston SL. The role of viruses in acute exacerbations of asthma. J Allergy Clin Immunol 2010;125:1178-87.

22 Ege MJ, Mayer M, Normand AC et al. Exposure to environmental microorganisms and childhood asthma. $N$ Engl J Med 2011;364:701-9.

23 Huang YJ, Marsland BJ, Bunyavanich S et al. The microbiome in allergic disease: current understanding and future opportunities 2017 PRACTALL Document of the American Academy of Allergy, Asthma \& Immunology and the European Academy of Allergy and Clinical Immunology. J Allergy Clin Immunol 2017;139:1099-110.

24 Chung KF. Airway microbial dysbiosis in asthmatic patients: a target for prevention and treatment? J Allergy Clin Immunol 2017;139:1071-81.

25 Sze MA, Hogg JC, Sin DD. Bacterial microbiome of lungs in COPD. Int J Chron Obstruct Pulmon Dis 2014;9:229-38.

26 Lipuma JJ. The changing microbial epidemiology in cystic fibrosis. Clin Microbiol Rev 2010;23:299-323.

27 Surette MG. The cystic fibrosis lung microbiome. Ann Am Thorac Soc 2014;11 Suppl 1:S61-5.

28 Whelan FJ, Heirali AA, Rossi L et al. Longitudinal sampling of the lung microbiota in individuals with cystic fibrosis. PLoS ONE 2017;12:e0172811.

29 Carmody LA, Zhao J, Kalikin LM et al. The daily dynamics of cystic fibrosis airway microbiota during clinical stability and at exacerbation. Microbiome 2015;3:12.

30 Cuthbertson L, Rogers GB, Walker AW et al. Respiratory microbiota resistance and resilience to pulmonary exacerbation and subsequent antimicrobial intervention. ISME J 2016;10:1081-91.

31 Cox MJ, Turek EM, Hennessy C et al. Longitudinal assessment of sputum microbiome by sequencing of the $16 \mathrm{~S}$ rRNA gene in noncystic fibrosis bronchiectasis patients. PLoS ONE 2017;12:e0170622.
32 Bernarde C, Keravec M, Mounier J et al. Impact of the CFTRpotentiator ivacaftor on airway microbiota in cystic fibrosis patients carrying a G551D mutation. PLoS ONE 2015;10:e0124124.

33 Molyneaux PL, Cox MJ, Willis-Owen SA et al. The role of bacteria in the pathogenesis and progression of idiopathic pulmonary fibrosis. Am J Respir Crit Care Med 2014;190:906-13.

34 Taylor SL, Woodman RJ, Chen AC et al. FUT2 genotype influences lung function, exacerbation frequency and airway microbiota in non-CF bronchiectasis. Thorax 2017;72:304-10.

35 Zhang Y, Moffatt MF, Cookson WO. Genetic and genomic approaches to asthma: new insights for the origins. Curr Opin Pulm Med 2012;18:6-13.

36 Land M, Hauser L, Jun SR et al. Insights from 20 years of bacterial genome sequencing. Funct Integr Genomics 2015;15:141-61.

37 Bentley SD, Parkhill J. Comparative genomic structure of prokaryotes. Annu Rev Genet 2004;38:771-92.

38 Couturier M, Bex F, Bergquist PL, Maas WK. Identification and classification of bacterial plasmids. Microbiol Rev 1988;52:375-95.

39 Redfield RJ. Do bacteria have sex? Nat Rev Genet 2001;2:634-9.

40 www.hpmcd.org/ [Accessed 10 October 2017].

41 Feigelman R, Kahlert CR, Baty F et al. Sputum DNA sequencing in cystic fibrosis: non-invasive access to the lung microbiome and to pathogen details. Microbiome 2017;5:20.

42 http://picrust.github.io/picrust/ [Accessed 10 October 2017].

43 Browne HP, Forster SC, Anonye BO et al. Culturing of 'unculturable' human microbiota reveals novel taxa and extensive sporulation. Nature 2016;533:543-6.

44 Dewhirst FE, Chen T, Izard J et al. The human oral microbiome. J Bacteriol 2010;192:5002-17.

45 Woo PC, Lau SK, Teng JL, Tse H, Yuen KY. Then and now: use of $16 \mathrm{~S}$ rDNA gene sequencing for bacterial identification and discovery of novel bacteria in clinical microbiology laboratories. Clin Microbiol Infect 2008;14:908-34.

46 Casadevall A, Pirofski LA. Microbiology: ditch the term pathogen. Nature 2014;516:165-6.

47 Siegel SJ, Weiser JN. Mechanisms of bacterial colonization of the respiratory tract. Annu Rev Microbiol 2015;69:425-44.

48 Patel R. New developments in clinical bacteriology laboratories. Mayo Clin Proc 2016;91:1448-59.

Address for correspondence: Professor W Cookson, Genomic Medicine, National Heart and Lung Institute, Imperial College London, Guy Scadding Building, Dovehouse Street, London SW3 6LY.

Email: w.cookson@imperial.ac.uk 